\title{
Side effects during chemotherapy predict tumour response in advanced colorectal cancer
}

\author{
B Schuell*,', T Gruenberger², GV Kornek', N Dworan', D Depisch ${ }^{3}$, F Lang $^{4}$, B Schneeweiss ${ }^{5}$ and \\ W Scheithauer'
}

'Division of Clinical Oncology, Department of Internal Medicine I, University Hospital, Waehringer Guertel I 8-20, Vienna A-1090, Austria; ${ }^{2}$ Department of Surgery, University Hospital, Waehringer Guertel 18-20, Vienna A-1090, Austria; ${ }^{3}$ Department of Surgery, Wr.Neustadt General Hospital, Corvinusring 3-5, Wr.Neustadt A-2700, Austria; ${ }^{4}$ Department of Surgery, Neunkirchen General Hospital, Peischinger Strasse 19, Neunkirchen A-2620, Austria;

${ }^{5}$ Department of Internal Medicine, Kirchdorf General Hospital, Kirchdorf an der Krems A-4560, Austria

To investigate whether a relationship between chemotherapy-associated adverse events and treatment efficacy exists, we have analysed the toxicity, objective response and survival data of 303 patients with advanced colorectal cancer. Patients were divided into two groups: the first with beneficial effect $(I, n=245)$, and the second with progressive disease $(I I, n=58)$. Differences in terms of incidence rates, type and severity of adverse events were analysed with univariate and multivariate models. The median number of side effects in group I was 6 vs 4 in group II $(O R=1.342 ; P=0.0001)$. An inverse correlation between disease control and treatment tolerance was confirmed when side effects were analysed according to severity and type of treatment-associated toxicities (haematological: $P=0.0005$ vs nonhaematological $P=0.0001$ ). When median survival was analysed according to the number of adverse events, it was $10(95 \% \mathrm{Cl}, 3-7), 16(14-18)$, and $18(16-20)$ months in case of $0-1,2-5$, and $\geqslant 6$ adverse events, respectively $(P=0.0 \mathrm{I})$. In conclusion, the results of this analysis suggest that occurrence of side effects during chemotherapy in advanced colorectal cancer is an independent and reliable prognostic indicator for response and survival.

British Journal of Cancer (2005) 93, 744-748. doi:I 0.I038/sj.bjc.6602783 www.bjcancer.com

Published online 13 September 2005

(c) 2005 Cancer Research UK

Keywords: colorectal cancer; side effects; chemotherapy; treatment efficacy

Colorectal cancer is one of the most common malignancies in the western world (Landis et al, 1999). Nearly one-third of all patients initially present with locally advanced, inoperable tumours and/or distant metastases (Williams et al, 1995; Midgley and Kerr, 1999), and approximately $50 \%$ of those having undergone potential curative surgery will ultimately develop recurrent disease. Median survival of these patients treated with best supportive care alone is approximately 6 months (Scheithauer et al, 1993); if treated with modern combination chemotherapy, median survival ranges from 17 to 21 months (Grothey et al, 2004). The effectiveness of conventional intravenous 5-fluorouracil (FU) given with or without biochemical modulators such as leucovorin (LV) or methotrexate, in fact, has been considerably improved with the recent development of a number of new, promising anticancer agents. These include the third generation 1,2-diaminocyclohexaneplatinum derivate oxaliplatin, and the topoisomerase I inhibitor irinotecan. When combined with FU/LV or the oral FU prodrug capecitabine, they seem to exert a synergistic effect which results in major improvement in overall response rate and progression-free survival, occasionally in overall survival (Grothey et al, 2004).

Potential prognostic markers determining response and/or survival have been described in numerous studies, and include a variety of clinical and laboratory parameters. In the largest series, a multivariate analysis of 3825 patients treated with FU-based

*Correspondence: Dr B Schuell; E-mail: birgit.schuell@meduniwien.ac.at Revised I August 2005; accepted 12 August 2005; published online 13 September 2005 chemotherapy, performance status, the number of metastatic sites, alkaline phosphatase, and the leukocyte count were identified as the most relevant parameters (Köhne et al, 2002).

As it concerns the relationship between toxicity incidence and treatment efficacy, only historical data are available. As indicated by Moertel in 1969, mild to moderate toxicity as objectively calibrated by leukocytopenia seems to result in optimal response to FU as well as its deoxyriboside FUDR (Moertel and Reiemeier, 1969; Ansfield, 1975). Apart from another small patient series with rectal cancer undergoing preoperative radiochemotherapy (Dahl et al, 1994), to our knowledge, no other conclusive data are available about treatment tolerance as a potential prognostic marker for response despite the fact that in $>80 \%$ of all patients receiving chemotherapy some kind of side effects (of course largely depending on which chemotherapy regimen is administered) will occur. Apart from use of inappropriate drug dosages in patients with impaired renal or hepatic function, presence of well-defined anticancer drug-specific risk factors such as DPD deficiency in case of FU-based chemotherapy (Diasio et al, 1988, 1998; Johnson et al, 1999; Milano et al, 1999), and to some extend gender (Stein et al, 1995; Sloan et al, 2002) and age >65 (Stein et al, 1995; Cascinu et al, 1996; Chiara et al, 1998; Popescu et al, 1999), the reason/mechanism is still unclear which predisposes patients to experience chemotherapy-related side effects.

As a result of uncertainties of a correlation between incidence rates and severity of side effects with the effectiveness of palliative chemotherapy in colorectal cancer, particularly as it concerns the novel more effective combination regimens, the present study was 
performed. In an attempt to answer this potentially important basic question, we have analysed the toxicity, objective response and survival data of 303 patients with advanced colorectal cancer who were consecutively treated in four prospective oxaliplatin- or irinotecan-based combination chemotherapy studies.

\section{PATIENTS AND METHODS}

\section{Patients}

A total of 303 patients with advanced colorectal cancer, who were entered in four different multicentre first- or second-line chemotherapy protocols between 1998 and 2001 were analysed (Scheithauer et al, 2001a, b, c, 2003). All patients had histologically confirmed metastatic or locally advanced/recurrent adenocarcinomas with bidimensionally measurable disease (defined as presence of at least one index lesion capable of two-dimensional measurement by computed tomography (CT) scan. Additional eligibility criteria in all four trials included age between 19 and 75 years, a World Health Organisation (WHO) performance status of two or less, and adequate bone marrow reserve, as well as renal and hepatic function. Adjuvant 5-FU-based chemotherapy and/or radiation was allowed if it was completed $\geqslant 6$ months before study entry. Within 2 weeks prior to initiating chemotherapy, all patients were assessed by physical examination, routine haematology and biochemistry analyses and CT-scans (or MRI) to define the extent of disease. Complete blood cell counts with platelet and differential counts were repeated weekly, and serum chemistries were determined at least once every course. All side effects, performance status, body weight, physical examination and subjective symptoms were recorded before each treatment course. Target lesions were reassessed by CT-scan or MRI every 8-12 weeks; objective response was evaluated according to WHO standard criteria.

\section{Chemotherapy}

The following six treatment regimens were administered to the 303 patients, who actively participated in four different trials: The first trial was a phase II study of front-line combination chemotherapy with raltitrexed $3 \mathrm{mg} \mathrm{m}^{-2}$ and oxaliplatin $130 \mathrm{mg} \mathrm{m}^{-2}$ both administered on day 1 every 3 weeks (Scheithauer et al, 2001a). In the second trial, the same dose regimen was administered to patients failing prior fluoropyrimidine/LV-based chemotherapy (Scheithauer et al, 2001b). The third trial was a randomised multicentre phase II trial of oxaliplatin plus irinotecan $v s$ raltitrexed as first-line treatment with a crossover design upon progression (Scheithauer et al, 2001c): patients randomised to the combination arm, were treated with oxaliplatin $85 \mathrm{mg} \mathrm{m}^{-2}$ and irinotecan $175 \mathrm{mg} \mathrm{m}^{-2}$ every 2 weeks. Patients randomised to the raltitrexed-arm received a dose of $3 \mathrm{mg} \mathrm{m}^{-2}$ given on day 1 every 3 weeks. The last trial was also a randomised multicentre phase II study of two different schedules of capecitabine plus oxaliplatin as first-line treatment in advanced colorectal cancer (Scheithauer et $\mathrm{al}, 2003)$. Half of the patients received oxaliplatin $130 \mathrm{mg} \mathrm{m}^{-2}$ on day 1 plus capecitabine $2000 \mathrm{mg}$ day on days $1-14$ every 3 weeks, in the other treatment arm patients received oxaliplatin $85 \mathrm{mg} \mathrm{m}^{-2}$ on days 1 and 14 combined with capecitabine $3500 \mathrm{mg} \mathrm{m}^{-2}$ day on days $1-7$ and $14-21$ every 4 weeks.

\section{Side effects}

Only chemotherapy-associated adverse reactions that occurred during the first 3 months of treatment were analysed. This was performed because (1) acute side effects generally occur within the first few courses, and (2) to avoid a possible selection bias by analysing fewer treatment courses in unresponsive patients. All except five patients received a minimum of 3 months chemotherapy
(98.3\%). Out of this five patients, two had a progressive disease after 2 months, two experienced severe toxicities and one patient refused further treatment after the first cycle of chemotherapy. Oxaliplatin-associated sensory neuropathy, which is cumulative in its nature, was not included in this analysis. Adverse events were graded according to WHO standard toxicity criteria. All side effects were first analysed in total, and then subdivided into haematological and nonhaematological side effects, as well as severe (grade $3 / 4$ ) and minor (grade $\leqslant 2$ ) toxicities.

\section{Statistical analyses}

Continuous covariates are described with mean and s.d. in the case of normally distributed data and with median, minimum and maximum for skewed covariates. Categorical variables are described with frequencies and percentages. Differences between the two interesting groups (complete remission (CR), partial remission (PR) and stable disease (SD) vs progressive disease (PD)) in terms of number of side effects, number of tumour sites, gender, age and chemotherapy regimen were examined with univariate and multiple logistic regression models always modelling the probability of experiencing CR; PR or SD vs PD. Number of side effects and all other categorisations of side effects (haematological and nonhaematological, WHO grade $>2$ and WHO grade $\leqslant 2$ ) as well as number of tumour sites are modelled as linear factors in logistic regression analyses, implying a constant odds ratio between consecutive variable values. All other scrutinised factors are taken into account as categorical variables, whereas categories male in case of gender, regimen, irinotecan plus oxaliplatin for chemotherapy, and age up to 65 years served as reference categories. All analyses were carried out using the statistical software package SAS (version 8.02, SAS Institute, Cary, NC, USA), $P$-values are two-sided and $P<0.05$ was considered statistically significant.

\section{RESULTS}

A total of 303 patients (190 men and 113 women) who received first- or second-line chemotherapy for advanced colorectal cancer in four different clinical trials between 1998 and 2001 were included in this analysis. The pretreatment characteristics of the study population(s) which were essentially similar across the four trials are summarised in Table 1. Patients were divided into two groups: the first group (group I) had a positive treatment effect, which included complete response, partial response and SD; the second group (group II) was rated progressive during chemotherapy. As shown in Table 2, out of the 303 patients, 245 were categorised in group I, and 58 patients in group II.

Out of the entire study population only six patients had no side effects, and 297 patients had experienced at least one adverse event. The median number of side effects (haematological and nonhaematological, all WHO grades) in group I was 6 (range, 012) compared to 4 (range, $0-13$ ) in case of treatment failure.

The chance to have CR, PR or SD, in fact, was noted to be increasing with an increasing number of side effects $(\mathrm{OR}=1.342$, $P<0.0001)$. Specifically, one additional side effect increases the odds of no PD by $34 \%$ (Table 3 ). The increasing incidence of side effects from $\mathrm{PD}$ to complete response showed a highly significant correlation $(P<0.0001)$ without overlapping 95\% confidence intervals from $\mathrm{PD}$ to $\mathrm{SD}$ onwards.

The observation of an inverse correlation of disease control with treatment tolerance was confirmed when side effects were analysed according to severity: in patients experiencing minor toxicities (WHO grade $\leqslant 2 ; n=284$ ), the median number of side effects was 3 (range, $0-10$ ) in case of treatment efficacy, and 2 (range, $0-7$ ) in nonresponders. The corresponding odds ratio in a univariate logistic regression model was statistically significant $(\mathrm{OR}=1.319$, 
Table I Selected pretreatment characteristics of the analyzed study population

\begin{tabular}{lcc}
\hline & No of patients, $\boldsymbol{n}$ (\%) \\
\hline Gender & 190 & $63 \%$ \\
Male & 113 & $37 \%$ \\
Female & & \\
Age & 66.5 & \\
Median & $38-79$ & \\
Range & & $46 \%$ \\
WHO performance status & 138 & $40 \%$ \\
0 & 123 & $14 \%$ \\
I & 42 & $64 \%$ \\
2 & & $36 \%$ \\
Primary site & 193 & \\
Colon & 110 & \\
Rectum & & \\
Sites of metastases & 218 & $38 \%$ \\
Liver & 75 & $62 \%$ \\
$\quad$ Lung & 99 & \\
Abdominopelvic mass & 118 & \\
Other & & \\
Number of metastatic sites & 116 & \\
Single & 187 & \\
Multiple & & \\
\hline
\end{tabular}

$P=0.002$ ). In patients with severe toxicities (WHO grade $>2$; $n=45$ ), responders had a median number of 2 side effects (range, $0-9$ ), whereas nonresponder only suffered from 1 (range, $0-7$; $\mathrm{OR}=1.372, P=0.0006$ ).

We then divided treatment-associated side effects into haematological and nonhaematological. In all, 252 patients $(83 \%)$ had haematological side effects and 289 patients (95\%) had at least one nonhaematological side effect. Patients with at least SD had significantly more $(P=0.0005)$ haematological side effects (median 3 , range $0-4$ ) than patients with tumour progression (median 1 , range $0-4)$. One haematological side effect more implies a 1.472 times higher odds to experience at least SD $(P=0.0005)$. The most common nonhaematological side effects (apart from the cumulative oxaliplatin-associated peripheral sensory neuropathy, which was excluded from this analysis), were diarrhoea, transient elevation of liver functional parameters and nausea/emesis. The median number of nonhaematological side effects of a responder were 4 (range, 0-9) vs 2 (range, $0-9$ ) in case of treatment failure, a difference that was again statistically significant $(P<0.0001$, OR: $1.40)$.

We subsequently analysed the correlation of the response status and side effects according to the various treatment regimens (Table 2). Of the patients (87\%) who received chemotherapy with oxaliplatin plus capecitabine were in group I (80 patients), and $13 \%$ (12 patients) had PD (group II). The median number of side effects in both groups was 6 (group I: range, $0-11$; group II: range, $2-8)$ : no difference was noted between the two groups for this particular combination regimen $(P=0.578, \mathrm{OR}$ : 1.07$)$. In all, 82 out

Table 2 Descriptive statistics

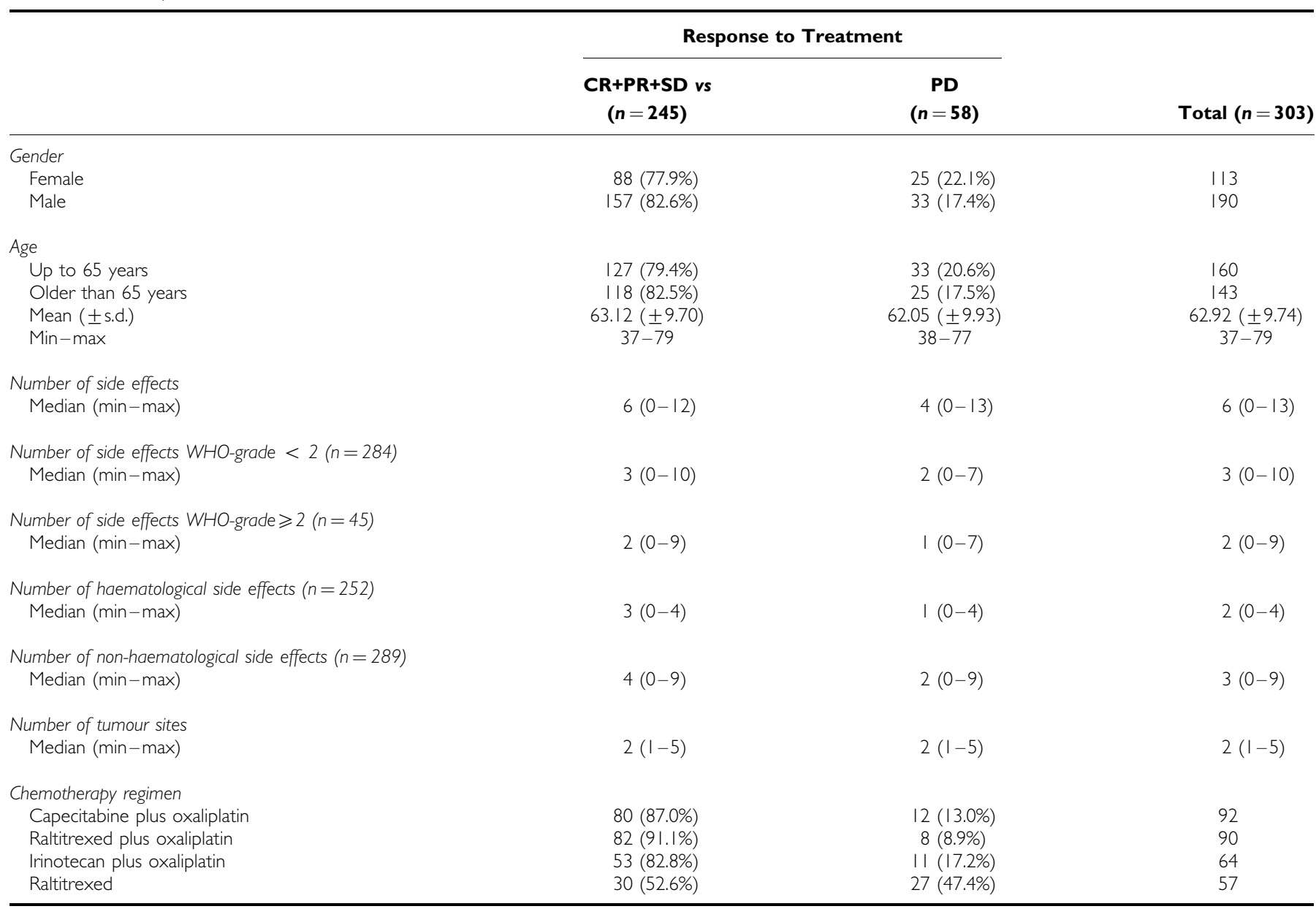

$\mathrm{CR}=$ complete response, $\mathrm{PR}=$ partial response, $\mathrm{SD}=$ stable disease, $\mathrm{PD}=$ progressive disease according to the World Health Organisation standard criteria. 
of 90 patients (91\%) who received raltitrexed plus oxaliplatin had an objective response or SD, and eight patients had PD. The median number of side effects in group I was 5 compared with 3.5 in group II, again a difference that did not reach the level of statistical significance $(P=0.0623$, OR: 1.46$)$. A total of 64 patients with metastatic colorectal cancer were treated with irinotecan plus oxaliplatin as first-line therapy. Of these patients, $83 \%$ were categorised in group I, and 11 patients in group II. In group I the median number of side effects was 8 as opposed to group II, where patients experienced a median number of 6 side effects $(P=0.028$, OR: 1.4). In all, 57 patients received raltitrexed as first-line chemotherapy. The median number of side effects was 5 in group I $(n=30)$, and in group II $(n=27)$ the median number of side effects was 3 ( $P=0.0038$, OR: 1.46$)$.

The median survival of all patients who had at least a stable disease was 19 months compared to 5.5 months in those with PD. When median survival was analysed according to the number of adverse events, it was 10 (95\%CI, 3-7), 16 (14-18), and 18 $(16-20)$ months in case of $0-1,2-5$ and $\geqslant 6$ AEs, respectively (log-rank test: $P=0.01$; Figure 1 ).

As indicated in Table 4, a multiple logistic regression model was used to assess the partial effects of number of side effects, number of tumour sites, gender, age and chemotherapy regimen in terms of treatment benefit. The number of side effects $(P<0.0001)$, the number of tumour sites $(P=0.003)$, and treatment with raltitrexed plus oxaliplatin $(P=0.0081)$ turned out to be significant prognostic factors. As it concerns gender and age, no statistical

Table 3 Univariate logistic regression analyses

\begin{tabular}{lcrrr}
\hline & Odds ratio & 95\% CI & P \\
\hline Number of side effects & 1.342 & $1.186-1.519$ & $<0.0001$ \\
Number of side effects WHO grade 2 & 1.319 & $1.107-1.572$ & 0.0020 \\
Number of side effects WHO grade $>2$ & 1.372 & $1.146-1.642$ & 0.0006 \\
Number of haematological side effects & 1.472 & $1.183-1.832$ & 0.0005 \\
Number of nonhaematological side effects & 1.398 & $1.182-1.654$ & $<0.0001$ \\
Gender (reference group: male) & 0.740 & $0.414-1.323$ & 0.3099 \\
Age (reference group: up to 65 years) & 1.226 & $0.689-2.184$ & 0.4880 \\
Number of tumour sites & 0.595 & $0.428-0.828$ & 0.0021 \\
\hline
\end{tabular}

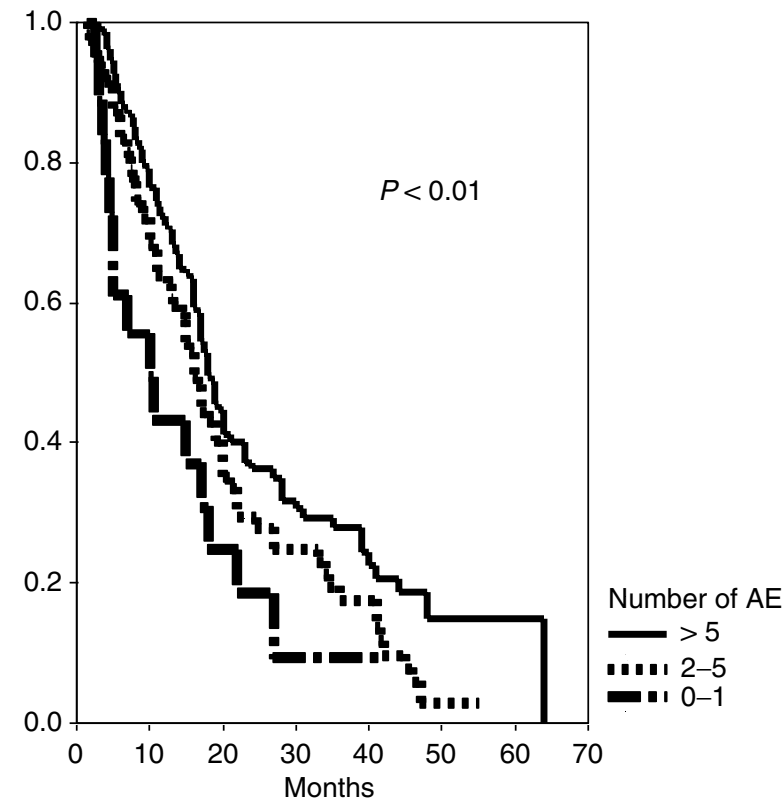

Figure I Median survival according to number of adverse events. difference was seen neither in the univariate nor in the multivariable analysis.

\section{DISCUSSION}

Administration of the same dose regimen of a specific anticancer drug or drug combination to a population of patients is likely to result in considerable variations of toxicities ranging from no side effect to potentially lethal events (Kuilenburg et al, 2001; Rothenberg et al, 2001). In our univariate and multivariable analyses the number of side effects associated with modern combination chemotherapy in advanced colorectal cancer turned out to be a significant independent prognostic factor; one additional adverse reaction, in fact, seems to increase the odds of response or SD by $34 \%$. Similarly, occurrence of side effects was found to correspond with an improved overall survival. The correlation between toxicity and therapeutic benefit was noted irrespective of the degree and type of toxicity, that is, nonhaematological $v s$ haematological. The latter observation seems important because haematological side effects are objectively measured toxicity parameters, which are not subject to a potential reporting bias. Variations of the correlation between toxicity and therapeutic benefit between the four treatment regimens are most likely to be related to the rather small study patient populations analysed, and especially the low rate of treatment failures in case of combination chemotherapy; this hypothesis is supported by the largest correlation of adverse events and response noted in the (least effective) raltitrexed control group in one of the studies.

It seems noteworthy that the correlation between toxicity and therapeutic benefit was not affected by gender and age. Differences in reporting subjective symptoms between men and women have been described in an analysis of 2448 patients who received FUbased chemotherapy for colorectal cancer (Sloan et al, 2002). Women experience toxicity more frequently and with more severity than men, whereby the gender variation could be related to the methylenetetrahydrofolate reductase C677T polymorphism (Van Rijnsoever et al, 2002), and/or the different levels of dihydropyrimidine dehydrogenase (Diasio, 1998; Milano et al, 1999). The current analysis also shows no difference between sexes in objective response rate or survival. The same is true for various otherwise rather divergent data about age and toxicity (Stein $e t a l$, 1995; Cascinu et al, 1996; Chiara et al, 1998; Popescu et al, 1999). Response and survival were not affected in these studies as they were in the current study, regardless of a difference in tolerance of 5-FU-based therapy between younger and older patients.

The results of our findings with the use of modern combination chemotherapy in advanced colorectal cancer are in agreement with an analysis of the relationship between the toxicity of FU $(n=176)$ and FUDR monotherapy $(n=203)$ to objective response rates in a comparable study population, which was published in 1969 (Moertel and Reiemeier, 1969). Fluoropyrimidine treatment to the point of mild or moderate leukocytopenia (1500-4500) resulted in a response rate of $22.7 v s 8.7$ and $15 \%$ in case of no

Table 4 Multiple logistic regression analysis

\begin{tabular}{lccr}
\hline & Odds Ratio & $\mathbf{9 5} \% \mathbf{C I}$ & \multicolumn{1}{c}{$\boldsymbol{P}$} \\
\hline Number of side effects & 1.349 & $1.167-1.559$ & $<0.0001$ \\
Gender (reference group: male) & 0.715 & $0.357-1.433$ & 0.3439 \\
Age (reference group: up to 65 years) & 1.556 & $0.799-3.033$ & 0.1939 \\
Number of tumour sites & 0.570 & $0.394-0.826$ & 0.0030 \\
Chemotherapy regimen & & & \\
$\quad$ Irinotecan plus oxaliplatin & 1.000 & & \\
$\quad$ Raltitrexed plus oxaliplatin & 4.271 & $1.457-12.521$ & 0.008 I \\
$\quad$ Raltitrexed & 0.526 & $0.198-1.395$ & 0.1966 \\
Capecitabine plus oxaliplatin & 1.697 & $0.654-4.406$ & 0.2770 \\
\hline
\end{tabular}


$(>4500)$ or more severe $(<1500)$ haematotoxicity. A randomised evaluation of a planned subtoxic FU dosage conducted by the Central Oncology Group has also demonstrated a significant inferiority in response rate when compared to a dose-producing mild to moderate toxicity (Ansfield, 1975). A third analysis we could identify in terms of correlating side effects with tumour response was published by Dahl et al (1994). The investigators described 159 patients who were treated with preoperative radiotherapy $(31.5 \mathrm{~Gy}$ in 18 fractions) for potentially resectable rectal adenocarcinoma. Patients were examined for a possible relationship between bowel toxicity manifested as diarrhoea and tumour size in the operative specimen as well as recurrence rate. Patients who required drugs for diarrhoea had significantly smaller tumours at surgery $(2.5 \mathrm{vs}$ $3.5 \mathrm{~cm}$ ). Furthermore, patients without significant radiation-induced diarrhoea had more recurrences (37.5 vs 14.3\%). The diseasespecific survival rate was also significantly better $(P=0.02)$ at 1.5 and 10 years in patients with diarrhoea WHO grades 3 and 4 . It was therefore concluded that a correlation between bowel sensitivity and tumour sensitivity to radiation might exist.

\section{REFERENCES}

Ansfield FJ (1975) A randomized phase III study of four dosage regimens of 5-fluorouracil. Proc Am Assoc Cancer Res 16: 224 (abstract)

Blum J, Jones S, Buzdar A (2001) Capecitabine (Xeloda) in 162 patients with paclitaxel-pretreated MBC: updated results and analyses of dose modification (abstract 693). Eur J Cancer 37(Suppl 6): S190 (abstract)

Cascinu S, Del Ferro E, Catalano G (1996) Toxicity and therapeutic response to chemotherapy in patients aged 70 years or older with advanced cancer. Am J Clin Oncol 19: $371-374$

Cassidy J, Twelves C, Van Cutsem E, Hoff P, Bajetta E, Boyer M, Bugat R, Burger U, Garin A, Graeven U, McKendric J, Maroun J, Marshall J, Osterwalder B, Perez-Manga G, Rosso R, Rougier P, Schilsky RL (2002) First-line oral capecitabine therapy in metastatic colorectal cancer: a favorable safety profile compared with iv 5-fluorouracil/leucovorin. Ann Oncol 13: 566-575

Chiara S, Nobile MT, Vincenti M, Lionetto R, Gozza A, Barzacchi MC, Sanguineti O, Repetto L, Rosso R (1998) Advanced colorectal cancer in the elderly: results of consecutive trials with 5-fluorouracil-based chemotherapy. Cancer Chemother Pharmacol 42: 336-340

Dahl O, Horn A, Mella O (1994) Do acute side-effects during radiotherapy predict tumor response in rectal carcinoma? Acta Oncol 33: 409-413

Diasio RB, Beavers TL, Carpenter JT (1988) Familial deficiency of dihydropyrimidine dehydrogenase. Biochemical basis for familial pyrimidinemia and severe 5-fluorouracil- induced toxicity. J Clin Invest 81: $47-51$

Diasio RB (1998) The role of dihydropyrimidine dehydrogenase (DPD) modulation in 5-FU pharmacology. Oncology (Huntingt) 12: 23-27

Grothey A, Sargent D, Goldberg R, Schmoll HJ (2004) Survival of patients with advanced colorectal cancer improves with the availability of fluorouracil, leucovorin, irinotecan, and oxaliplatin in the course of treatment. J Clin Oncol 22: 1209-1214

Johnson MR, Hageboutros A, Wang L, High L, Smith JB, Diasio RB (1999) Life-threating toxicity in a dihydropyrimidine dehydrogenase-deficient patient after treatment with topical 5-fluorouracil. Clin Cancer Res 5: 2006-2011

Köhne CH, Cunningham D, Di Costanzo F, Glimelius B, Blijham G, Aranda E, Scheithauer W, Rougier P, Palmer M, Wils J, Baron B, Pignatti F, Schoffski P, Micheel S, Hecker H (2002) Clinical determinants of survival in patients with 5 -fluorouracil based treatment for metastatic colorectal cancer: results of a multivariate analyses of 3825 patients. Ann Oncol 13: $308-317$

Kuilenburg AB, Muller EW, Haasjes J, Meinsma R, Zoetekouw L, Waterham HR (2001) Lethal outcome of a patient with a complete dihydropyrimidine dehydrogenase (DPD) deficiency after administration of 5fluorouracil: frequency of the common IVS14 + 1G $>$ A mutation causing DPD deficiency. Clin Cancer Res 7: 1149-1153

Landis SH, Murray T, Bolden S (1999) Cancer statistics 1999. CA Cancer J Clin 49: 8-13

Midgley R, Kerr D (1999) Colorectal cancer. Lancet 353: 391 - 399
In conclusion, the results of this analysis of over 300 patients with advanced colorectal cancer suggest that occurrence of side effects during modern combination chemotherapy in advanced colorectal cancer is an independent prognostic indicator of response. Although these data might have to be confirmed for more commonly used fluoropyrimidine combination regimens (such as FOLFOX or FOLFIRI) the correct interpretation of the present and the few other available corresponding results would already be available: as perfectly articulated by Moertel in the fluoropyrimidine monotherapy era about 35 years ago: 'To achieve the most favourable results you must play the piper, but not too much' (Moertel and Reiemeier, 1969). Patients should receive adequate drug dosages, which may warrant individualised dose escalations. At no price, however, treatmentassociated side effects should be too severe and thus interfere with the patients' quality-of-life. As demonstrated in recent trials, dose reductions in patients experiencing severe toxicity do not lead to compromised efficacy (Cassidy et al, 2002; Blum et al, 2001).
Milano G, Etienne M, Pierrefite V, Barberi-Heyob M, Deporte-Fety R, Renee N (1999) Dihydropyrimidine dehydrogenase deficiency and fluorouracilrelated toxicity. Br J Cancer 79: 627-630

Moertel CG, Reiemeier RJ (1969) Advanced Gastrointestinal Cancer. Clinical Management and Chemotherapy. New York: Harper \& Row Pubs Inc.

Popescu RA, Norman A, Ross PJ, Parikh B, Cunninham D (1999) Adjuvant or palliative chemotherapy for colorectal cancer in patients 70 years or older. J Clin Oncol 17: 2412-2418

Rothenberg ML, Meropol NJ, Poplin EA, van Cutsem E, Wadler S (2001) Mortality associated with irinotecan plus bolus fluorouracil/ leucovorin: summary findings of an independent panel. J Clin Oncol 19: $3801-3807$

Scheithauer W, Kornek GV, Raderer M, Schuell B, Schmid K, Kovats E, Schneeweiss B, Lang F, Lenauer A, Depisch D (2003) Randomized multicenter phase II trial of two different schedules of capecitabine plus oxaliplatin as first-line treatment in advanced colorectal cancer. J Clin Oncol 21: $1307-1312$

Scheithauer W, Kornek GV, Raderer M, Ulrich-Pur H, Fiebiger W, Gedlicka C, Schull B, Brugger S, Schneeweiss B, Lang F, Lenauer A, Depisch D (2001a) Randomized multicenter phase II trial of oxaliplatin plus irinotecan versus raltitrexed as first-line treatment in advanced colorectal cancer. J Clin Oncol 20: 165 - 172

Scheithauer W, Kornek GV, Schuell B, Ulrich-Pur H, Penz M, Raderer M, Lang F, Schneeweiss B, Lenauer A, Depisch D (2001b) Second-line treatment with oxaliplatin+raltitrexed in patients with advanced colorectal cancer failing fluoropyrimidine/leucovorin-based chemotherapy. Ann Oncol 12: 709-714

Scheithauer W, Kornek GV, Ulrich-Pur H, Penz M, Raderer M, Salek T, Haider K, Kwasny W, Depisch D (2001c) Oxaliplatin plus raltitrexed in patients with advanced colorectal carcinoma. Cancer 91: 1264-1271

Scheithauer W, Rosen H, Kornek GV, Sebesta C, Depisch D (1993) Randomized comparison of combination chemotherapy plus supportive care with supportive care alone in patients with metastatic colorectal cancer. Br Med J 306: $752-756$

Sloan JA, Goldberg RM, Sargent DJ, Vargas-Chanes D, Nair S, Cha SS, Novotny PJ, Poon MA, O'Connell MJ, Loprinzi CL (2002) Women experience greater toxicity with fluorouracil-based chemotherapy for colorectal cancer. J Clin Oncol 20: $1491-1498$

Stein BN, Petrelli NJ, Douglass HO, Driscoll DL, Arcangeli G, Meropoe NJ (1995) Age and sex are independent predictors of 5-fluorouracil toxicity. Analysis of a large scale phase III trial. Cancer 74: 11-17

Van Rijnsoever M, Grieu F, Elsaleh H, Joseph D, Iacopetta B (2002) Characterization of colorectal cancers showing hypermethylation at multiple CpG islands. Gut 51: 797-802

Williams NS, Northover JMA, Arnott SJ (1995) Colorectal tumors. In Oxford Textbook of Oncology, In Peckham M, Pinedo H, Veronesi U (eds) Oxford, UK: Oxford University Press, pp 1133-1168 